\title{
CENTRAL NERVOUS SYSTEM RELAPSE OF MULTIPLE MYELOMA
}

\author{
Maria Qubtia ${ }^{1}$, Muhammad Umer Nasir ${ }^{2}$, Memoona Mian ${ }^{3}$, Abdul Hameed $^{1}$ \\ ${ }^{1}$ Department of Medical Oncology, Shaukat Khanum Memorial Cancer Hospital and Research Centre, Lahore, \\ Pakistan, ${ }^{2}$ Department of Radiology, Shaukat Khanum Memorial Cancer Hospital and Research Centre, Lahore, \\ Pakistan, ${ }^{3}$ Department of Diagnostic Radiology, University of Arkansas for Medical Sciences, Little Rock, USA \\ Received: 2 September 2018 / Accepted: 26 February 2019
}

\begin{abstract}
Multiple myeloma (MM) is a plasma cell disorder primarily involving bone marrow. Extramedullary involvement is less common, with central nervous system (CNS) myelomatosis being a rare entity and such presentation carries extremely dismal prognosis. We present case of a 40 years old male with MM who was initially treated with 6 cycles of Cyclophosphamide, Thalidomide and Dexamethasone resulting in complete response. 2 years later he presented with CNS myelomatosis and scrotal involvement and was initially treated with Bortezomib and dexamethasone, cranial irradiation and intrathecal Methorexate, Cytarabine, Hydrocortisone (TRIO IT), along with radical orchiectomy and testicular radiation during the course of treatment. However, after initial response his disease showed clinical and radiological progression after 4 months of therapy. He was switched to high dose Methotrexate (HD-MTX) with TRIOIT and later Lenalidamide and dexamethasone (Len/dex) was added to the above regimen. He continued to show good clinical response but his cytology remained persistently positive, therefore, HD-MTX was discontinued in the later course of treatment. Subsequently he was started on best supportive care only, when his neurological status deteriorated further. He survived almost 9 months after a diagnosis of CNS myelomatosis. Patients with multiple myeloma, presenting with neurological symptoms should always be investigated for the possibility of CNS MM. CNS relapse is a fatal disease with poor prognosis. Recommended treatment must include a systemic anti-MM regimen that crosses the BBB (ideally Immunomodulatory drugs (IMiDs) IMiDs-dexamethasone based therapy), CNS irradiation and intrathecal chemotherapy.
\end{abstract}

Key words: Multiple myeloma, central nervous system myelomatosis, therapy

\section{Introduction}

Multiple myeloma (MM) is a clonal disorder of plasma cells, mainly involving the bone marrow ${ }^{[1]}$ Extramedullary involvement of MM is seen in approximately $7 \%$ of the patients. ${ }^{[2]}$ Central nervous system (CNS) involvement in $\mathrm{MM}$ is uncommon. Amongst its possible presentations, leptomeningeal dissemination of MM causing myelomatous meningitis (MyM), also termed CNS-myelomatosis, is one of the rarest complications of $\mathrm{MM}^{[3]}$ with only a handful of reported cases and is characterised by the presence of neoplastic plasma cells in the cerebrospinal fluid (CSF). The incidence is reported to be approximately $1 \%{ }^{[4]}$ of MM patients with the median overall survival of $<6$ months. ${ }^{[5]}$ Treatment of MyM is seldom curative and essentially

Correspondence: Dr. Maria Qubtia, Department of Medical Oncology, Shaukat Khanum Memorial Cancer Hospital and Research Centre, Peshawar, Pakistan.

Email: marythecopt12@yahoo.com palliative, with attempted preservation of neurologic function. ${ }^{[6]}$ We present a case of treated $\mathrm{MM}$ who presented with isolated CNS relapse in the form of leptomeningeal disease after 2 years of achieving complete response (CR).

\section{Case Report}

A 40-year-old gentleman was diagnosed in 2012 with MM after a left humeral pathological fracture with tissue biopsy positive for plasma cell disorder, International Scoring System-II (marrow plasmacytosis $20 \%$, M protein $28.7 \mathrm{~g} / \mathrm{dl}$ in gamma region, urine protein electrophoresis negative, beta 2 microglobulin $4 \mathrm{mg} / \mathrm{dL}$ and disseminated lytic lesions). Baseline whole spine imaging revealed multilevel osseous involvement with a significant compression fracture of the L5 vertebral body without spinal cord compromise at any level [Figures 1 and 2].

The patient completed 6 cycles of cyclophosphamide weekly, thalidomide once daily and dexamethasone (Dex) days 1-4, 


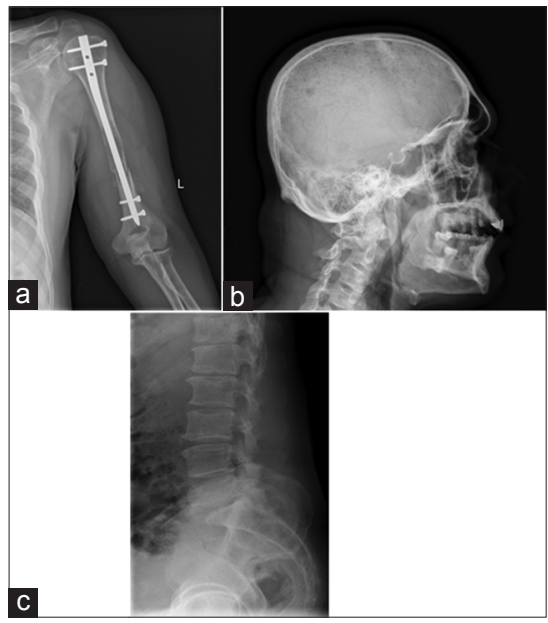

Figure 1: (a) Frontal projection of the left humerus shows internal intramedullary nail fixation of distal diaphyseal fracture background lytic lesion with endosteal scalloping. (b) Lateral projection of skull shows punched-out lytic lesions. (c) Lateral lumbar radiograph demonstrates that wedge compression fracture of L5 vertebral body is noted with background osteopenia

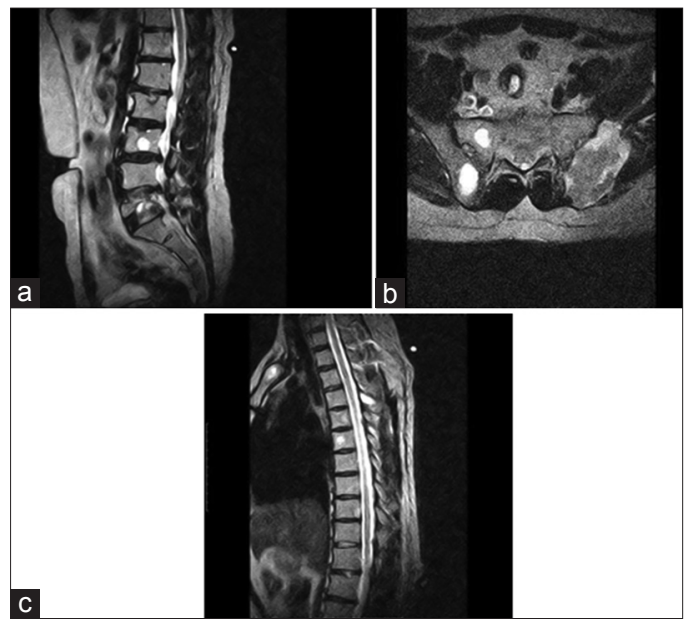

Figure 2: (a) T2-weighted sagittal magnetic resonance imaging (MRI) of lumbar spine shows T2 hyperintense well-defined lesion in L3 vertebral body and compression fracture of L5 vertebral body. (b) Axial T2-weighted image shows bilateral sacral disease involvement. (c) Sagittal T2-weighted MRI of thoracic spine demonstrates T2 hyperintense lesions of similar morphology in thoracic spine and manubrium sterni

9-12 (Cyclophosphamide, thalidomide, and dexamethasone) every 4 weeks and Zoledronic acid every 4 weeks and achieved CR in September 2012. Upfront high-dose therapy and autologous stem cell transplant were suggested to the patient, but he opted to be kept on surveillance only. In the meantime, the patient continued to receive monthly pamidronate.
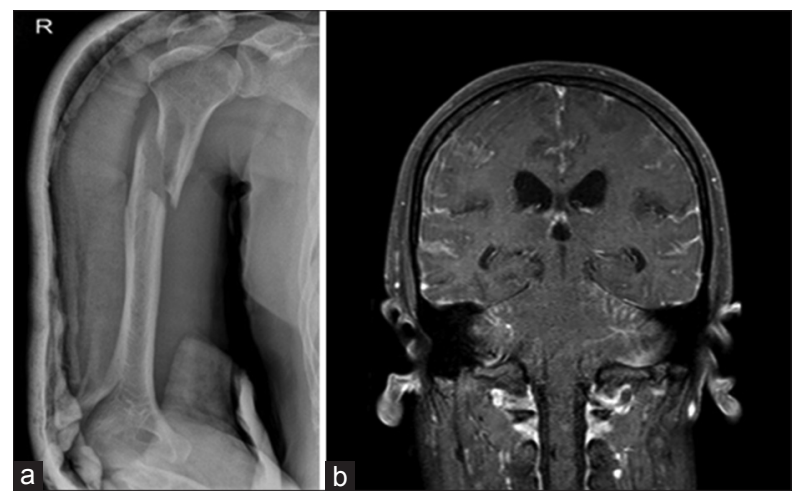

Figure 3: (a) AP projection of the right humerus shows an oblique complete fracture involving the proximal diaphysis with slight overriding of fracture fragments and displacement. (b) Coronal post-contrast magnetic resonance imaging of brain reveals diffuse supra- and infra-tentorial leptomeningeal enhancement with mild ventriculomegaly

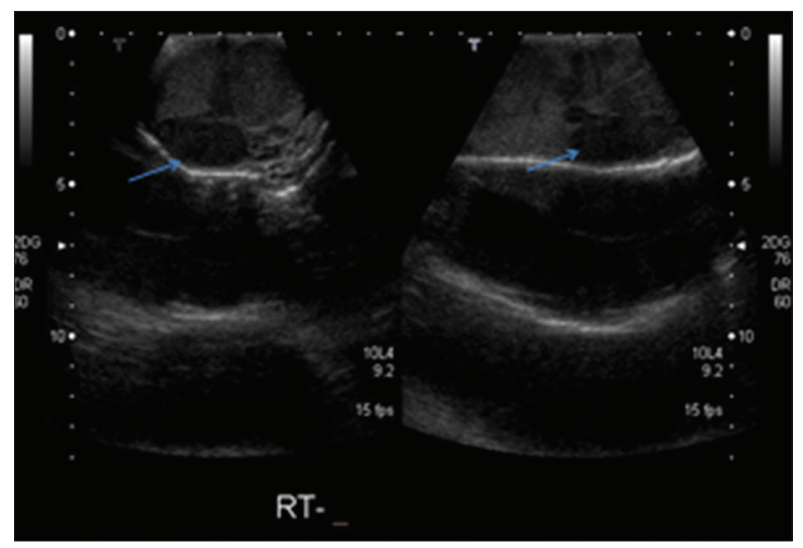

Figure 4: Transverse and longitudinal ultrasound image of the right testis shows enlargement and heterogonous parenchymal echotexture with hypoechoic lesions (arrow)

In September 2014, the patient presented to the emergency room with a history of fall, a pathological fracture of the right humerus, dizziness, poor oral intake and altered sensorium. Magnetic resonance (MR) brain revealed diffuse leptomeningeal enhancement with mild communicating hydrocephalus concerning for infectious, inflammatory or MyM [Figure 3]. CSF revealed high protein $(151 \mathrm{mg} / \mathrm{dl}), \mathrm{LDH}$ and normal glucose. CSF cytology was positive for atypical plasma cells. His repeat bone marrow biopsy and blood workup at that point did not show any evidence of systemic disease. Flow cytometry showed approximately $96 \%$ of total cellular events characterised as CD45+/-, CD38++ and CD138+, confirming isolated CNS involvement by MM. 


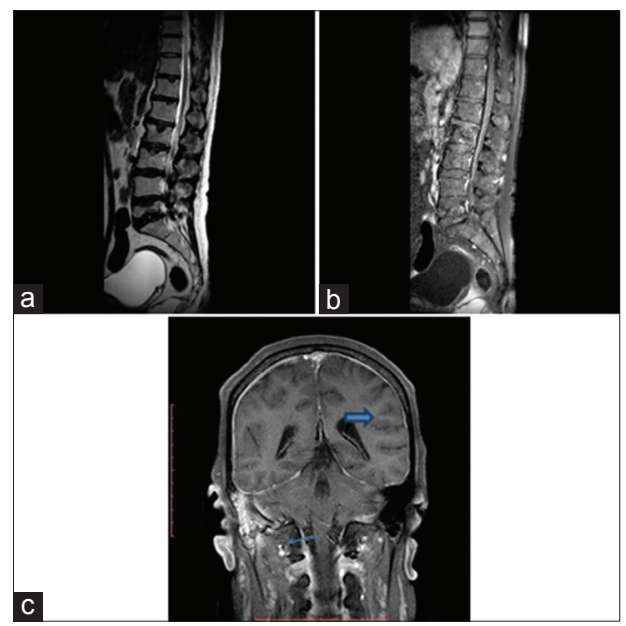

Figure 5: (a and b) T2 and post-contrast T1-weighted sagittal magnetic resonance imaging of lumbar spine shows interval decrease in the T2 high signal lesion in L3 vertebral body lesion. However, interval development of leptomeningeal enhancement along the spinal cord is suggestive of progressive meningeal myelomatous disease. (c) T1 post-contrast coronal images through brain show pachymeningeal $(\rightarrow$ ) and leptomeningeal $(\rightarrow)$ enhancement

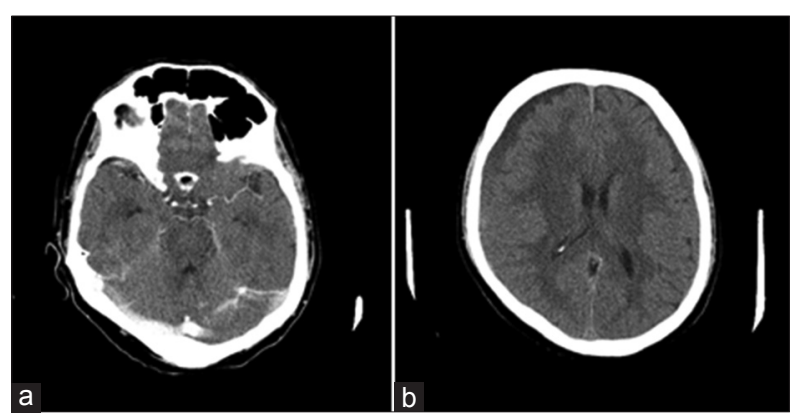

Figure 6: Contrast-enhanced computed tomography scan of the brain shows persistent meningeal enhancement along the basilar cisterns (a) with the right-sided subdural effusion causing subtle mass effect on the ipsilateral cerebral hemisphere (b)

Therefore, the patient was started on methotrexate, cytarabine and hydrocortisone given intrathecally, (TRIO-IT), and received cranial irradiation followed by bortezomib on days 1, 4, 8 and 11 and Dex on days 1-4 (Bordex) every 3 weeks. He showed clinical improvement in his higher mental function after 2 weeks of therapy. The patient was subsequently noticed to have right testicular swelling [Figure 4] and right radical orchiectomy was performed with myelomatous involvement on histopathology. Later on, palliative radiotherapy to testicular area and humerus was offered as well. Clinical, cytological and radiological evaluation after 2 cycles of Bordex showed partial response.

In December 2014, clinical, cytological and radiological CNS disease progression was noted [Figure 5]. As a consequence, high-dose methotrexate every 3 weeks along with TRIO-IT was started. Lenalidomide (Len) was added to above regimen in view of disease progression. After completing 3 cycles of high-dose methotrexate and 1 cycle of Len and Dex, good clinical response was noted; however, CSF cytology was still positive and computed tomography showed persistent meningeal enhancement [Figure 6]. Therapy apart from TRIO-IT was stopped. In April 2015, he developed deterioration in neurological status and showed persistently positive cytology for atypical plasma cells. The patient was deemed to have refractory disease, and hence, active oncological treatment was withheld and was kept on best supportive care. The patient passed away almost 9 months after CNS disease relapse.

\section{Discussion}

Leptomeningeal myelomatous involvement is very rare. The clinical presentations of CNS MM can be variable and can range from impaired cognition, headache, vomiting drowsiness, confusion, limb weakness, cranial nerve palsies, seizures and urinary incontinence. ${ }^{[6]}$ The differential diagnosis of such a clinical presentation in MM includes hypercalcemia, hyperviscosity, spinal cord compression, CNS infection and peripheral neuropathy associated with immune-modulatory agents, amyloid deposition ${ }^{[6]}$ and, rarely, POEMS syndrome.

The recommended workup for patients should include brain imaging, particularly MR imaging (MRI) brain, CSF analysis and complete metabolic screening to rule out differential diagnoses as mentioned above. Clonal characterisation of the atypical cells by cytology and flow cytometry is required. Protein measurement including intrathecal M-component by electrophoresis and immune fixation is also helpful to reach a diagnosis. Fluorescent in situ hybridisation testing for evaluation of the cytogenetic abnormalities should be performed, where available. For those cases, where MRI is suggestive of CNS involvement and CSF analysis including flow cytometry is inconclusive, surgical biopsy should be considered. 
Since CNS involvement by MM is rarely seen, there are no consensus guidelines on the management. ${ }^{[7]}$ A study done by Usmani et al. has also highlighted an unmet need in this subset of patients with high risk, relapsed or refractory MM. ${ }^{[8]}$ Therapy of CNS extramedullary myeloma (EMM) is difficult, needs both systemic and CNS-specific therapy. Systemic therapy for CNS EMM should include drugs that may cross the blood-brain barrier (BBB). Durable responses have been reported using thalidomide. ${ }^{[9]}$ Pomalidomide has demonstrated a good penetration of the BBB and has shown efficacy in treating disease with CNS manifestations. ${ }^{[10,11]}$ Bendamustine can be used as well. Recommended treatment must include a systemic anti-MM regimen that crosses the BBB (ideally an IMiD-dexamethasone-based therapy), CNS irradiation and intrathecal chemotherapy. ${ }^{[5]}$

There is a need for prospective trials to establish clear guidelines about the management of this disease; however, this may be relatively difficult due to a small number of patients presenting with this complication.

\section{Conclusion}

Patients with MM, presenting with neurological symptoms, should always be investigated for the possibility of CNS MM. CNS relapse is a fatal disease with poor prognosis. Recommended treatment must include a systemic anti-MM regimen that crosses the BBB (ideally an IMiD-dexamethasone-based therapy), CNS irradiation and intrathecal chemotherapy.

\section{Conflict of Interest}

The authors declare that they have no conflict of interest.

\section{References}

1. Dimopoulos MA, Terpos E. Multiple myeloma. Ann Oncol 2010;21 Supp1 7:vii143-50.
2. Usmani SZ, Heuck C, Mitchell A, et al. Extramedullary disease portends poor prognosis in multiple myeloma and is over-represented in high-risk disease even in the era of novel agents. Haematologica 2012;97:1761-7.

3. Fassas AB, Muwalla F, Berryman T, et al. Myeloma of the central nervous system: Association with high-risk chromosomal abnormalities, plasmablastic morphology and extramedullary manifestations. Br J Haematol 2002;117: 103-8.

4. Chen CI, Masih-Khan E, Jiang H, et al. Central nervous system involvement with multiple myeloma: Long term survival can be achieved with radiation, intrathecal chemotherapy, and immunomodulatory agents. Br J Haematol 2013;162:483-8.

5. Nieuwenhuizen L, Biesma DH. Central nervous system myelomatosis: Review of the literature. Eur J Haematol 2008;80:1-9.

6. Katodritou E, Terpos E, Kastritis E, et al. Lack of survival improvement with novel anti-myeloma agents for patients with multiple myeloma and central nervous system involvement: The greek myeloma study group experience. Ann Hematol 2015;94:2033-42.

7. Yutaka H, Mariko Y, Shinichiro O, et al. Thalidomide for the treatment of leptomeningeal multiple myeloma. Eur J Haematol 2006;76:358-9.

8. Li Z, Qiu Y, Personett D, et al. Pomalidomide shows significant therapeutic activity against CNS lymphoma with a major impact on the tumor microenvironment in murine models. PLoS One 2013;8:e71754.

9. Mussetti A, Dalto S, Montefusco V. Effective treatment of pomalidomide in central nervous system myelomatosis. Leuk Lymphoma 2013;54:864-6.

10. Chamberlain MC, Glantz M. Myelomatous meningitis. Cancer 2008;112:1562-7.

11. Abdallah AO, Atrash S, Shahid Z, et al. Patterns of central nervous system involvement in relapsed and refractory multiple myeloma. Clin Lymphoma Myeloma Leuk 2014;14:211-4.

\section{Authorship Contributions}

Concept and Design: MQ, MUN, MM, AH; Data Collection and interpretation: MQ, MUN, AH; Literature review and writing: $\mathrm{MQ}, \mathrm{MM}, \mathrm{AH}$; Manuscript approval: MQ, MUN, MM, AH 